\title{
Gain Scheduling PID Controller via Least Squares Optimization *
}

\author{
Rafael F. Q. Magossi ${ }^{*, * *}$ Ricardo Q. Machado ${ }^{* *}$ Vilma A. Oliveira $^{* *}$ \\ * CEFET/RJ - Campus Nova Friburgo \\ Av. Gov. Roberto Silveira, 1900 \\ Nova Friburgo, Rio de Janeiro, Brasil (e-mail: rafael.magossi@cefet-rj.br) \\ ** Universidade de São Paulo \\ Av. Trabalhador Sancarlense, 400 \\ São Carlos, São Paulo, Brasil (e-mail: rquados@usp.br, voliveira@usp.br)
}

\begin{abstract}
Usually the industrial plants are modelled in a nominal situation and then the controller is designed based on it. However, the dynamics of industrial plants are generally dependents of parameters, which can change or even can not be properly estimate until a final implementation. In this paper, it is proposed an adaptive PID controller to work around with parameter variations and pursue a nominal designed condition. The problem is modeled as a linear system, where the gains of the PID controller are the variables of interest, then the stability constraints are imposed on the system. As the linear system to be solved is over-determined, the least squares method was used and it was implement as a quadratic optimization problem. Besides, the stability in case to switch the controller during the operation is also covered and a set of numerical examples are presented including a simulation on PSIM software.

Resumo: Normalmente, as plantas industriais são modeladas em uma situação nominal e, em seguida, o controlador é projetado a considerando. No entanto, a dinâmica das plantas industriais são geralmente dependentes de parâmetros, que podem mudar ou mesmo não ser adequadamente estimados até a implementação final. Neste artigo, é proposto um controlador PID adaptativo para lidar com as variações de parâmetros buscando a condição nominal projetada. O problema é modelado como um sistema linear, onde os ganhos do controlador PID são as variáveis de interesse, então as restrições de estabilidade são impostas ao sistema. Como o sistema linear a ser resovido é sobredeterminado, foi utilizado o método dos mínimos quadrados implementado como um problema de otimização quadrática. Além disso, a estabilidade em caso de troca do controlador durante a operação também é tratada e um conjunto de exemplos numéricos é apresentado incluindo uma simulação no PSIM.
\end{abstract}

Keywords: Quadratic programming; adaptive control; boost converter; stabilizing set; Toeplitz matrix.

Palavras-chaves: Programação quadrática; controle adaptativo; conversor boost; conjunto estabilizante; matriz Toeplitz.

\section{INTRODUCTION}

Widespread in the industry, the proportional+integral+derivative (PID) controllers are the most common solution implemented and in the literature it is possible find several tuning methods (Åström and Hägglund, 1995; Keel and Bhattacharyya, 2008). New PID controller tuning techniques have appeared to consider the problem of partial or complete unknown of the plant model (Bhattacharyya et al., 2009; Khadraoui et al., 2013). Analytical approaches used to specify stability margins considering achievable performance with PI controller have also been considered for different plants (Li, 2013; Alzate and Oliveira, 2016; Díaz-Rodríguez and Bhattacharyya, 2016). The results include the computation of stabilizing sets, gain and phase margins using the generalization of Hermite-Biehler theorem for polynomials, and as a matter of fact, attractiveness to work with polynomials has increased due to its simplicity (Keel and Bhattacharyya, 2008; Bhattacharyya et al., 2009).

\footnotetext{
* This study was supported in part by the Coordenação de Aperfeiçoamento de Pessoal de Nível Superior (CAPES) under grant 001, and by São Paulo Research Foundation (FAPESP) under grants 2016/21120-2 and 2016/25017-1.
}

In industry, the dynamics of its plants are generally not completely known and subject to changes. To address the problem of parameter variations and uncertainties in the plant, the robust and adaptive control approach can be used. Each approach has its inevitable merits and disadvantages (Bhattacharyya et al., 2009). In robust control, the designed controller can make the system remain stable even in the presence of plant uncertainty. Due to modeling errors, the nominal performance of the system is often not guaranteed and ensuring the robustness of system performance can result in an increasingly conservative design (Bhattacharyya et al., 2009; Huang et al., 2002).

On the other hand, within the framework of adaptive control, theory and applications for self-tuning have been extensively reported in research papers and books. For adaptive controllers, performance specifications are maintained by performing selftuning of controllers when the system deviates from nominal. Controller stability with self-tuning is still a challenge for researchers, despite advances in the area (Huang et al., 2002).

An way to work around with self-tuning is using the gain scheduling controller strategy, which is one of the most com- 
monly used solutions in the industry to overcome the nonlinear process characteristics by adapting controller gains relative to local operating bands (Blanchett et al., 2000). Solutions for gain scheduling using fuzzy logic were presented in Zhao et al. (1993) and Blanchett et al. (2000).

Therefore, in this paper we propose a simple adaptation procedure for PID controller in sense of gain scheduling scheme using a polynomial equation, which allows to solve the problem as a linear algebraic system under linear constraints with a quadratic optimization criterion. Following this introduction, in Section 2 we present a lemma as preliminaries to Section 3 , where the proposed procedure is presented. In Section 4 we presented numerical examples and in Section 5 some final remarks.

\section{PRELIMINARIES}

In this section, we present a lemma which relates a polynomial product to the resulting polynomial coefficients, which is the basis for the main results.

Lemma 1. Consider the product between two polynomials in $s$ as

$$
\sum_{k=0}^{f_{1}} \alpha_{k} s^{k} \sum_{k=0}^{f_{2}} \beta_{k} s^{k}=\sum_{k=0}^{f_{1}+f_{2}} \gamma_{k} s^{k}
$$

where $f_{1}, f_{2} \in \mathbb{N}, \alpha_{k}, \beta_{k}$ e $\gamma_{k} \in \mathbb{R}$. Thus, $V v=\nu$, in which,

$V=\left[\begin{array}{ccccc}\alpha_{0} & & & & \\ \alpha_{1} & \alpha_{0} & & & 0 \\ \alpha_{2} & \alpha_{1} & \alpha_{0} & & \\ \vdots & \ddots & \ddots & \ddots & \\ \vdots & & \ddots & \ddots & \alpha_{0} \\ \alpha_{f_{1}} & & \ddots & \ddots & \alpha_{1} \\ & \ddots & \ddots & & \alpha_{2} \\ & & \ddots & & \vdots \\ & & & \ddots & \alpha_{f_{1}-1} \\ 0 & & & & \alpha_{f_{1}}\end{array}\right], v=\left[\begin{array}{c}\beta_{0} \\ \beta_{1} \\ \beta_{2} \\ \vdots \\ \beta_{f_{2}-1} \\ \beta_{f_{2}}\end{array}\right]$ and $\nu=\left[\begin{array}{c}\gamma_{0} \\ \gamma_{1} \\ \gamma_{2} \\ \vdots \\ \gamma_{f_{1}+f_{2}-1} \\ \gamma_{f_{1}+f_{2}}\end{array}\right]$

with $V \in \mathbb{R}^{\left(f_{1}+f_{2}+1\right) \times\left(f_{2}+1\right)}, v \in \mathbb{R}^{\left(f_{2}+1\right) \times 1}$ and $\nu \in$ $\mathbb{R}^{\left(f_{1}+f_{2}+1\right) \times 1}$.

The proof of Lemma 1 is direct using finite induction and is omitted.

Remark 1. Matriz $V$ in Lemma 1 has a Toeplitz matrix form and we use the notation $V=\mathcal{T}\left(\left[\alpha_{0} \cdots \alpha_{f_{1}}\right]\right)$.

To exemplify the use of Lemma 1, let's consider the product

$$
\left(9 s^{2}+2 s+1\right)(-2 s+7)=-18 s^{3}+59 s^{2}+12 s+7 .
$$

Applying Lemma 1, we can write

$$
\left[\begin{array}{ll}
1 & 0 \\
2 & 1 \\
9 & 2 \\
0 & 9
\end{array}\right]\left[\begin{array}{r}
7 \\
-2
\end{array}\right]=\left[\begin{array}{r}
7 \\
12 \\
59 \\
-18
\end{array}\right] \text {. }
$$

With Lemma 1, the main results are presented in the next section.

\section{MAIN RESULTS}

Consider a control loop with unity feedback as shown in Fig. 1 with $r(t)$ the reference signal, $u(t)$ the plant input, $y(t)$ the plant output and $e(t)$ the error between the reference and the output.

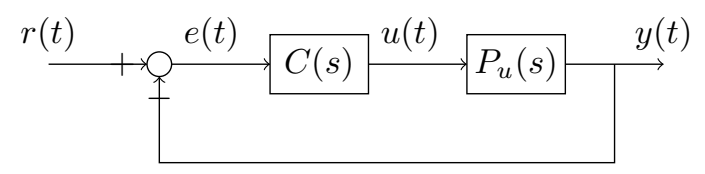

Figure 1. Control loop with unity feedback.

Let

$$
\begin{array}{r}
P_{u}(s)=: \frac{N_{u}(s)}{D(s)}=\frac{\sum_{k=0}^{n_{u}} g_{k} s^{k}}{\sum_{k=0}^{d_{p}} b_{k} s^{k}}, \\
C(s)=: \frac{N_{c}(s)}{s}=\frac{\sum_{k=0}^{n_{c}} c_{k} s^{k}}{s}
\end{array}
$$

be the plant and the controller, respectively. For simplicity, dropping some function notation, the closed-loop transfer function relating $r(t)$ to $y(t)$ in Fig. 1 is

$$
M_{F}(s)=\frac{C(s) P_{u}(s)}{1+C(s) P_{u}(s)}=\frac{N_{c} N_{u}}{s D+N_{c} N_{u}} .
$$

Suppose that for a nominal plant denoted $P_{u_{n}}(s)$, a controller denoted $C_{n}(s)$ is designed, then

$$
M_{F_{n}}(s)=\frac{N_{c_{n}} N_{u_{n}}}{s D_{n}+N_{c_{n}} N_{u_{n}}} .
$$

The objective is to keep the closed-loop transfer function close to the transfer function obtained with the nominal controller, this implies that it is sufficient

$$
\begin{aligned}
& N_{c} N_{u}=N_{c_{n}} N_{u_{n}}, \\
& N_{c} N_{u}=N_{c_{n}} N_{u_{n}}+s\left(D_{n}-D\right) .
\end{aligned}
$$

Thus write

$$
\begin{aligned}
N_{c_{n}} N_{u_{n}} & =\sum_{k=0}^{o} q_{k} s^{k} \\
N_{c_{n}} N_{u_{n}}+s\left(D_{n}-D\right) & =\sum_{k=0}^{l} p_{k} s^{k}
\end{aligned}
$$

with $o=n_{u}+n_{c}$ and $l=\max \left(n_{u}+n_{c}, d_{p}+1\right)$. Lemma 1 is the basis of the proposed approach, as well as the following assumption

Assumption 1. $n_{u}+n_{c}=\max \left(n_{u}+n_{c}, d_{p}+1\right)$.

The controller of interest is a PID, described by

$$
C(s)=\frac{k_{d} s^{2}+k_{p} s+k_{i}}{s} .
$$

Thus, using Lemma 1 and Assumption 1 we can write the following system of linear algebraic equations

$$
\left[\begin{array}{l}
G \\
G
\end{array}\right] \mathbf{x}=\left[\begin{array}{l}
\mathbf{q} \\
\mathbf{p}
\end{array}\right]
$$

in which $\mathbf{p}=\left[\begin{array}{lllll}p_{0} & p_{1} & \cdots & p_{l-1} & p_{l}\end{array}\right]^{T}$ and $\mathbf{q}=\left[\begin{array}{lllll}q_{0} & q_{1} & \cdots & q_{o-1} & q_{o}\end{array}\right]^{T}$ are obtained with (10) and (9), repectively, and 


$$
G=\left[\begin{array}{ccc}
g_{0} & 0 & 0 \\
g_{1} & g_{0} & 0 \\
g_{2} & g_{1} & g_{0} \\
g_{3} & g_{2} & g_{1} \\
g_{4} & g_{3} & g_{2} \\
& \vdots & \\
g_{n_{u}} & g_{n_{u}-1} & g_{n_{u}-2} \\
0 & g_{n_{u}} & g_{n_{u}-1} \\
0 & 0 & g_{n_{u}}
\end{array}\right], \quad \mathbf{x}=\left[\begin{array}{c}
k_{i} \\
k_{p} \\
k_{d}
\end{array}\right]
$$

Note that (12) is a over-determined linear system and one way to solve it, is to use the least square solution, which is explored in the next subsections.

\subsection{Solution via least squares method}

Let $\mathbf{c}=\left[\mathbf{q}^{T} \mathbf{p}^{T}\right]^{T}$ and $Q=\left[G^{T} G^{T}\right]^{T}$. As the linear system $Q \mathbf{x}=\mathbf{c}$ is over-determined, one can find a solution that best fits the system by minimizing some criterion. It is used the one that produces the lowest Euclidian norm of the gains of the controller, which follows:

$$
\mathbf{x}=\left(Q^{T} Q\right)^{-1} Q^{T} \mathbf{c}=Q^{+} \mathbf{c}
$$

where $Q^{+}=\left(Q^{T} Q\right)^{-1} Q^{T}$ is the inverse pseudo-matrix of Moore-Penrose. We can rewrite the optimal solution as a linear system

$$
\hat{Q} \mathbf{x}=\hat{B}
$$

in which $\hat{Q}=: Q^{T} Q$ and $\hat{B}=: Q^{T} \mathbf{c}$.

Although the least squares method find the optimal gain, it is possible that the gain found does not satisfy the stability criterion. In order to work around this problem, the next subsection gives an alternative to the least squares method, modelling it as a quadratic optimization problem with constraints.

\subsection{Solution via quadratic optimization with constraints}

The quadratic optimization problem subject to linear constraints can be formulated as follows

subject to

$$
\min _{\mathbf{x}} \frac{1}{2}\|Q \mathbf{x}-\mathbf{c}\|^{2},
$$

$$
R \mathbf{x} \leq \mathbf{w}
$$

where $R$ is a matrix and $\mathbf{w}$ a vector of appropriate dimensions.

The objective function (16) can be rewritten as

$$
\begin{aligned}
\min _{\mathbf{x}} \frac{1}{2}\|Q \mathbf{x}-\mathbf{c}\|^{2} & =\min _{\mathbf{x}} \frac{1}{2}(Q \mathbf{x}-\mathbf{c})^{T}(Q \mathbf{x}-\mathbf{c}) \\
& =\min _{\mathbf{x}} \frac{1}{2}\left(\mathbf{x}^{T} Q^{T} Q \mathbf{x}-2 \mathbf{x}^{T} Q^{T} \mathbf{c}+\mathbf{c}^{T} \mathbf{c}\right) .
\end{aligned}
$$

As $\mathbf{c}^{T} \mathbf{c}$ is fixed, according to Coleman and $\mathrm{Li}$ (1996), it is sufficient to solve the following quadratic optimization problem

$$
\min _{\mathbf{x}} \frac{1}{2} \mathbf{x}^{T} H \mathbf{x}+\mathbf{f}^{T} \mathbf{x}
$$

using

$$
H=Q^{T} Q, \quad \mathbf{f}=-Q^{T} \mathbf{c} .
$$

The matrix $R$ and $\mathbf{w}$ are constraints on the PID gains to guarantee stability which can be obtained by the Routh-Hurwitz criterion for low-order systems. However, for higher-order systems, the constraints on the PID gains can be obtained using the generalization of Hermite-Biehler theorem, which always results in linear constraints for fixed $k_{p}$ gain (Datta et al., 2000).

The application of the proposed optimization can be usefully in a gain scheduling strategy. Such that, in the case of operating conditions changes, the controller gains can be adapted. However, it is necessary to guarantee the stability of the gain scheduling controller.

\subsection{Gain scheduling controller stability}

Consider the following switched closed loop system matrices in standard state space form as

$$
\left[\begin{array}{c|c}
A_{i} & B_{i} \\
\hline C_{i} & D_{i}
\end{array}\right]
$$

where $i \in\{1, \cdots, n\}$ is the index that selects the subsystems arbitrarily according to the operation condition. The global stability of the switched system (21) can be guaranteed by the following definition and theorem taken from Skafidas et al. (1999).

\section{Definition 1. Let}

$$
Z_{1}=Z_{1}^{T}, Z_{2}=Z_{2}^{T}, \cdots, Z_{n}=Z_{n}^{T}
$$

matrices given. It is said that the set $\left\{Z_{1}, Z_{2}, \cdots, Z_{n}\right\}$ is strictly complete if for any $x_{0} \in \mathrm{R}^{j} /\{0\}, j \in \mathrm{N}^{+}, x_{0}^{T} Z_{i} x_{0}<$ $0, \forall i \in\{1, \cdots, n\}$.

Theorem 2. System (21) is asymptotically stable if exists $P=$ $P^{\prime}>0$ such that

$$
Z_{i} \triangleq A_{i}^{T} P+P A_{i}, \forall i \in\{1, \cdots, n\}
$$

is strictly complete.

Let the realization of $M_{F_{n}}(s)$ be

$$
\left[\begin{array}{l|l}
A_{1} & B_{1} \\
\hline C_{1} & D_{1}
\end{array}\right]
$$

and considering the optimal gains returned by (16), denote the realization of $M_{F}(s)$ as

$$
\left[\begin{array}{l|l}
A_{2} & B_{2} \\
\hline C_{2} & D_{2}
\end{array}\right]
$$

then using Theorem 2, solve for $P$ the following set of linear matrix inequalities (LMI)

$$
\left\{\begin{array}{l}
A_{1}^{T} P+P A_{1}<0, \\
A_{2}^{T} P+P A_{2}<0 .
\end{array}\right.
$$

Therefore, to ensure stability in the gain scheduling procedure, it is sufficient that for every plant and its adapted controller there exists a $P$ matrix common to all systems. In the next subsection, numerical examples are presented.

\section{NUMERICAL EXAMPLES}

In this section, we present some numerical examples. In the first, we consider a third order system designed to a nominal condition with the controller obtained by the least square method without any constraints. In the second, we consider a boost converter and used the least square method formulated as a quadratic programming with linear stability constraints. Finally, we consider again the boost converter example to show the effect of changing the controller gains under a scheduling strategy. 
Example 1. Consider a plant with the following nominal transfer function:

$$
P_{u_{n}}(s)=\frac{10 s^{2}+5 s+1}{100 s^{3}+50 s^{2}+10 s+1}
$$

and a designed PID controller:

$$
C_{n}(s)=\frac{s^{2}+15 s+6}{s} .
$$

The closed loop transfer function is

$$
M_{F_{n}}(s)=\frac{10 s^{4}+155 s^{3}+136 s^{2}+45 s+6}{110 s^{4}+205 s^{3}+146 s^{2}+46 s+6} .
$$

Next, assume that the actual plant is

$$
P_{u}(s)=\frac{8 s^{2}+7 s+1}{120 s^{3}+30 s^{2}+15 s+1} .
$$

First find that $n_{u}=2, d_{p}=3$ and $n_{c}=2$ which satisfy $n_{u}+n_{c}=\max \left(n_{u}+n_{c}, d_{p}+1\right)$. Then, using Lemma 1 the following matrix are found

$$
\begin{aligned}
& G=\left[\begin{array}{lll}
1 & 0 & 0 \\
7 & 1 & 0 \\
8 & 7 & 1 \\
0 & 8 & 7 \\
0 & 0 & 8
\end{array}\right], \quad \mathbf{q}=\left[\begin{array}{c}
6 \\
45 \\
136 \\
155 \\
10
\end{array}\right], \\
& \mathbf{p}=\left[\begin{array}{c}
6 \\
45 \\
131 \\
175 \\
-10
\end{array}\right], \quad \mathbf{c}=\left[\begin{array}{ll}
\mathbf{q}^{T} & \mathbf{p}^{T}
\end{array}\right]^{T} .
\end{aligned}
$$

The PID gains are obtained by solving the following algebraic linear system

$$
\left[\begin{array}{lll}
1 & 0 & 0 \\
7 & 1 & 0 \\
8 & 7 & 1 \\
0 & 8 & 7 \\
0 & 0 & 8 \\
11 & 0 & 0 \\
7 & 1 & 0 \\
8 & 7 & 1 \\
0 & 8 & 7 \\
0 & 0 & 8
\end{array}\right]\left[\begin{array}{l}
k_{i} \\
k_{p} \\
k_{d}
\end{array}\right]=\left[\begin{array}{c}
6 \\
45 \\
136 \\
155 \\
10 \\
6 \\
45 \\
131 \\
175 \\
-10
\end{array}\right] .
$$

Using a Moore-Penrose pseudo-inversa, the PID solution with the least Euclidian norm can be obtained as

$$
\begin{aligned}
& k_{i}=1.7720, \\
& k_{p}=18.7364, \\
& k_{d}=0.8239 .
\end{aligned}
$$

In Fig. 2, a comparison between the responses is shown. An improvement in the response is evident when performing an action to return the closed loop system to its nominal operating condition.

Remark 2. Notice that the PID controller can be considered in its practical form with a derivative filter term:

$$
C(s):=\frac{k_{d} s^{2}+k_{p} s+k_{i}}{s(s+\tau)}
$$

where $\tau$ is previously fixed.

It may happen that the controller obtained by the least squares optimization procedure is not able to stabilize the feedback system. It is critical, therefore, to restrict the controller gains

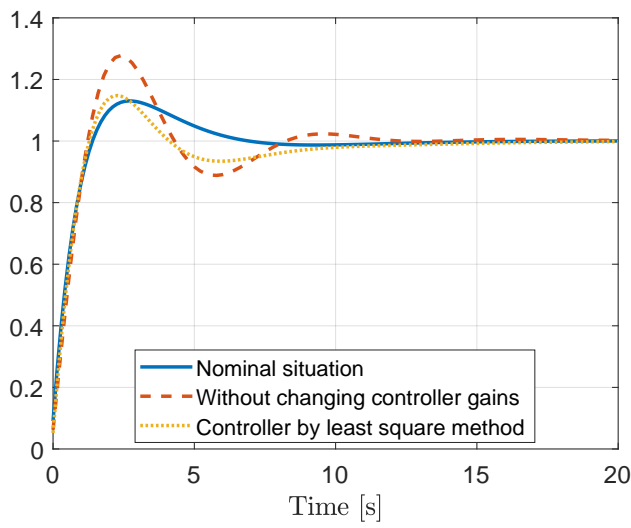

Figure 2. Step response of the system comparing designed nominal situation, altered plant with nominal controller and modified plant with controller by least square method without stability constraints.

to belong to the set of stabilizing controllers. The next example consider the adaptation under the quadratic optimization with stability constraints.

Example 2. To illustrate the proposed adaptation method, an application example follows, but using the constraint on the gains for the nominal case. Consider the boost converter shown in Fig. 3 with double loop control for regulating the output voltage.

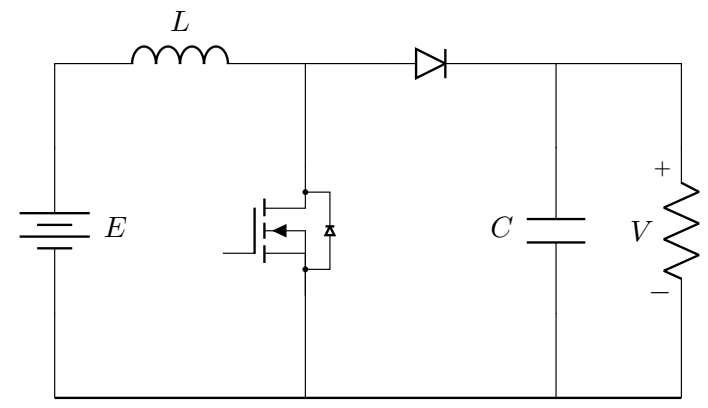

Figure 3. Ideal boost converter.

The output voltage $V$ is regulated indirectly by the drive input current. For the internal loop, a controller of the sliding mode type and in the external loop a PI controller are used. The simulation schematic on PSIM is shown in Fig. 4. The transfer function of the inner loop, according to Alzate and Oliveira (2016), is

$$
P_{u}(s)=\frac{-L V^{2} s+R E^{2}}{C R E V s+2 E V} .
$$

Considering the nominal condition $L=1 \mathrm{mH}, C=1000 \mu \mathrm{F}$, $V=100 \mathrm{~V}, E=10 \mathrm{~V}$ e a load $R=50 \Omega$, the nominal plant is

$$
P_{u_{n}}(s)=\frac{-10 s+5000}{50 s+2000} \text {. }
$$

From the nominal plant, the following PI controller was obtained

$$
C(s)=0.005+\frac{73}{s},
$$

such that the closed-loop system has the following transfer function 


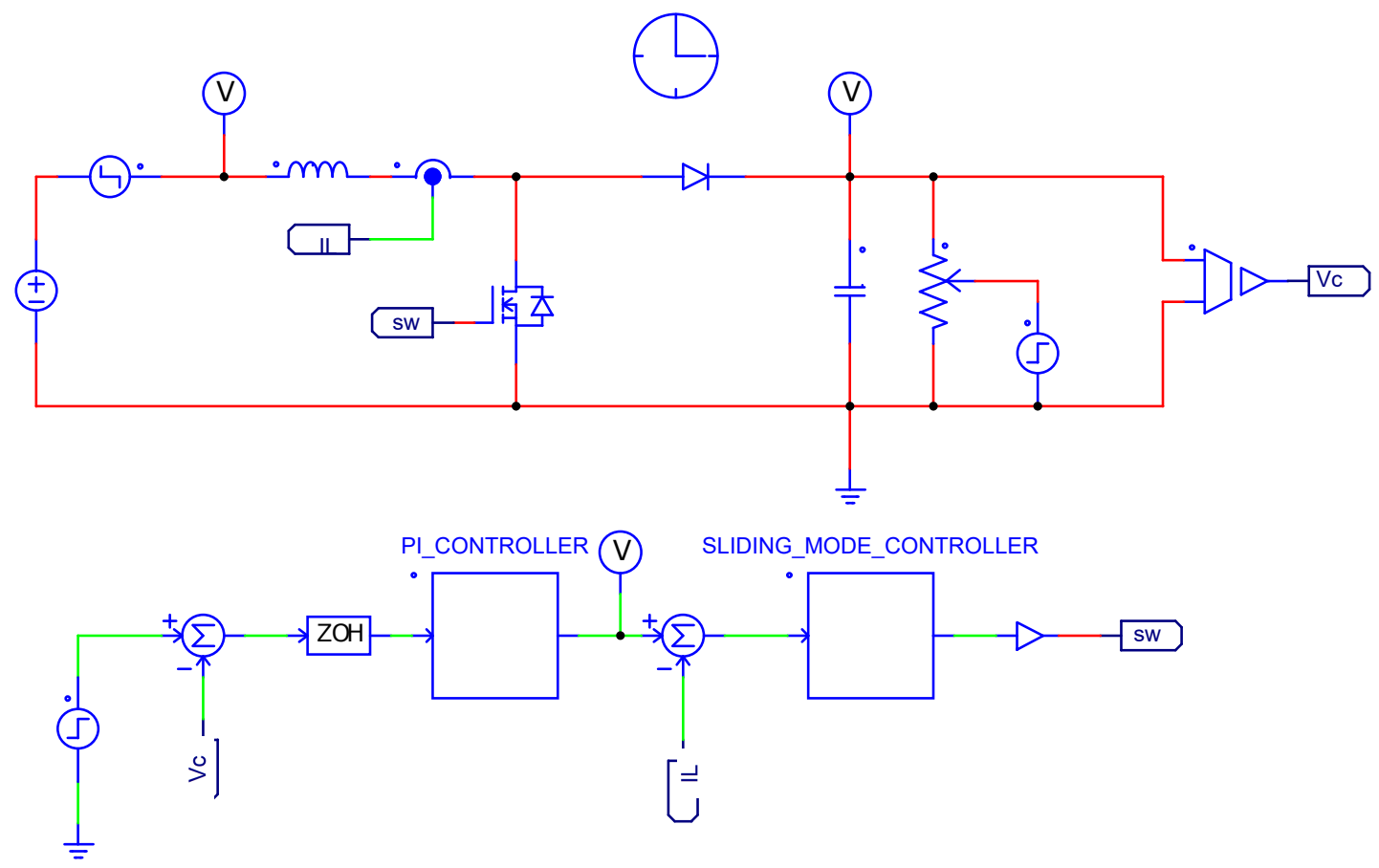

Figure 4. Double loop control of a boost converter in PSIM software.

$$
M_{F_{n}}=\frac{-0.01 s^{2}-495 s+250000}{49.99 s^{2}+1505 s+250000} .
$$

Suppose the load in the system changes to $R=100 \Omega$ and a voltage drop in power supply of $5 \mathrm{~V}$ occurs. In this case, the plant becomes

$$
P(s)=\frac{-10 s+2500}{50 s+1000} .
$$

It is then desired to adjust the controller again so that the nominal condition is approximated. Then we first identify $n_{u}=$ $d_{p}=n_{c}=1$, which satisfies $n_{u}+n_{c}=\max \left(n_{u}+n_{c}, d_{p}+1\right)$. From (36), with Lemma 1 we obtain

$$
\begin{aligned}
G & =\left[\begin{array}{cc}
2500 & 0 \\
-10 & 2500 \\
0 & -10
\end{array}\right], \\
q & =\left[\begin{array}{c}
250000 \\
-495 \\
-0.01
\end{array}\right], \quad p=\left[\begin{array}{c}
250000 \\
505 \\
-0.01
\end{array}\right]
\end{aligned}
$$

and

$$
\left[\begin{array}{cc}
2500 & 0 \\
-10 & 2500 \\
0 & -10 \\
\hline 2500 & 0 \\
-10 & 2500 \\
0 & -10
\end{array}\right]\left[\begin{array}{l}
k_{i} \\
k_{p}
\end{array}\right]=\left[\begin{array}{c}
250000 \\
-495 \\
-0.01 \\
\hline 250000 \\
505 \\
-0.01
\end{array}\right] \text {. }
$$

To ensure that the system is stable, it is necessary to impose some restrictions on the $k_{i}$ and $k_{p}$. From the determination of the stabilizing set, these restrictions can be obtained. In the case of this system, according to Alzate and Oliveira (2016), these constraints are

$$
\begin{aligned}
& k_{p}<\frac{C E R V}{L V^{2}}=5 \\
& k_{i}<\left(\frac{E^{2} R}{L V^{2}}\right) k_{p}+\left(\frac{2 E}{L V}\right)=250 k_{p}+100, \\
& k_{i}>0 .
\end{aligned}
$$

Since these constraints are linear and the cost function is quadratic, we used the interior point algorithm in Matlab to obtain $k_{i}=100$ and $k_{p}=0,4020$. The step responses for all the controllers are shown in Fig. 5, where there is a significant improvement when compared to the situation in which the controller does not change.

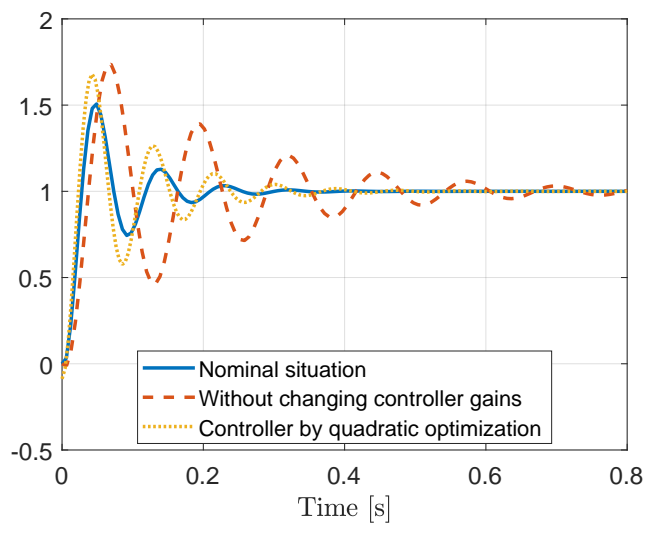

Figure 5. Step responses of the system comparing the designed nominal situation, altered plant with nominal controller and modified plant with the optimized controller under linear stability constraints.

Example 3. In order to consider a gain scheduling scenario in which the controller switch to a new one when a parameter variation is detected, we had to verify the stability for the switched system. Thus, considering the nominal closed loop system, from (35) the controllable canonical realization is

in which

$$
\left[\begin{array}{l|l}
A_{1} & B_{1} \\
\hline C_{1} & D_{1}
\end{array}\right]
$$

$$
A_{1}=\left[\begin{array}{cr}
0 & -5001.0 \\
1.0 & -30.106
\end{array}\right], B_{1}=\left[\begin{array}{c}
1.0 \\
0
\end{array}\right] \text {, }
$$




$$
C_{1}=\left[\begin{array}{ll}
-9.896 & 5299.9
\end{array}\right], D_{1}=-0.00020004 \text {. }
$$

and the controllable canonical realization of the closed loop system with the optimized controller is

such that

$$
\left[\begin{array}{l|l}
A_{2} & B_{2} \\
\hline C_{2} & D_{2}
\end{array}\right]
$$

$$
\begin{aligned}
& A_{2}=\left[\begin{array}{cr}
0 & -5437.1 \\
1.0 & -21.857
\end{array}\right], B_{2}=\left[\begin{array}{c}
1.0 \\
0
\end{array}\right], \\
& C_{2}=\left[\begin{array}{ll}
2.0193 & 5868.4
\end{array}\right], D_{2}=-0.087428 .
\end{aligned}
$$

Then, solving (25) it returns

$$
P=\left[\begin{array}{cc}
0.00020315 & -0.0038167 \\
-0.0038167 & 0.9998
\end{array}\right]
$$

and, therefore, the switched system is stable under the gain scheduling strategy. The gain scheduling was implemented in PSIM as shown in Fig 4 and the result is shown in Fig 6.

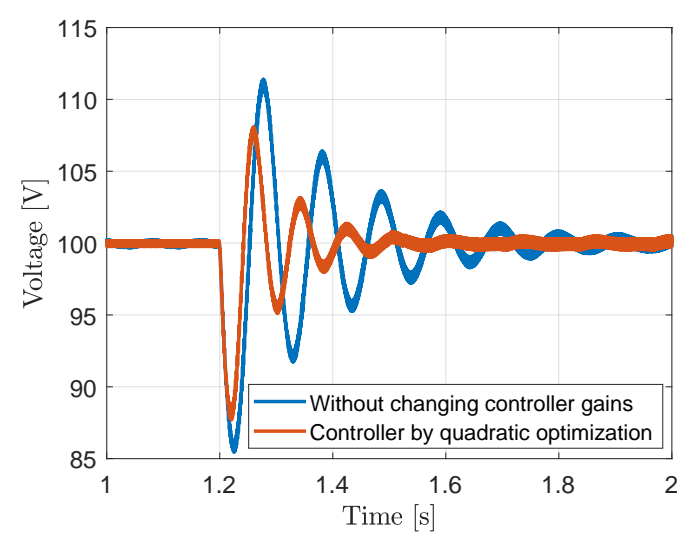

Figure 6. Simulation performed in PSIM with a gain scheduling strategy. The blue line is the closed loop response with boost converter parameters changes and the nominal controller, whereas the red line is the closed loop response obtained by changing the controller gains to the obtained by the least squares method under a quadratic programming optimization.

In Fig 6, the gain scheduling strategy showed that changing the PID controller gains is better than not.

\section{FINAL REMARKS}

In this paper it was presented a gain scheduling PID controller. The problem lies in finding the gains $k_{p}, k_{i}$, and $k_{d}$ so that the performance of the system feedback to a nominal condition is pursued under varying plant operating conditions. The PID gains are obtained using a quadratic optimization method with constraints due to its simplicity and consolidation in the literature. The problem is formulated as an optimization of linear algebraic system. As the resulting linear system is overdetermined, the least squares method was used in a quadratic optimization form. The stability for a situation in which the controllers can change during the operation was also considered. The numerical examples showed that the use of the adaptation procedure (for offline or in a gain scheduling operation) gives better results than not changing the nominal controller. Future works include expanding the results to resonant controllers.

\section{REFERENCES}

Alzate, R. and Oliveira, V.A. (2016). Multiobjective design of PI controllers with applications. In 2016 IEEE Conference on Control Applications (CCA), 203-214. IEEE, Buenos Aires.

Åström, K. and Hägglund, T. (1995). PID Controllers: Theory, Design, and Tuning. Instrument Society of America, Research Triangle Park, 2nd edition.

Bhattacharyya, S.P., Datta, A., and Keel, L.H. (2009). Linear Control Theory: Structure, Robustness, and Optimization. Automation and Control Engineering. CRC Press, Boca Raton, 1 edition.

Blanchett, T.P., Kember, G.C., and Dubay, R. (2000). PID gain scheduling using fuzzy logic. ISA Transactions, 39(3), 317 325.

Coleman, T.F. and Li, Y. (1996). A reflective newton method for minimizing a quadratic function subject to bounds on some of the variables. SIAM Journal on Optimization, 6(4), 10401058.

Datta, A., Ho, M., and Bhattacharyya, S.P. (2000). Structure and Synthesis of PID Controllers. Advances in Industrial Control. Springer-Verlag, London.

Díaz-Rodríguez, I.D. and Bhattacharyya, S.P. (2016). PI controller design in the achievable gain-phase margin plane. In IEEE 55th Conference on Decision and Control, 4919-4924. IEEE, Las Vegas.

Huang, H.P., Roan, M.L., and Jeng, J.C. (2002). On-line adaptive tuning for PID controllers. IEE Proceedings Control Theory and Applications, 149(1), 60-67.

Keel, L.H. and Bhattacharyya, S.P. (2008). Controller synthesis free of analytical models: Three term controllers. IEEE Transactions on Automatic Control, 53(6), 1353-1369.

Khadraoui, S., Nounou, H.N., Nounou, M.N., Datta, A., and Bhattacharyya, S.P. (2013). A measurement-based approach for designing reduced-order controllers with guaranteed bounded error. International Journal of Control, 86(9), 1586-1596.

$\mathrm{Li}, \mathrm{K}$. (2013). PID tuning for optimal closed-loop performance with specified gain and phase margins. IEEE Transactions on Control Systems Technology, 21(3), 1024-1030.

Skafidas, E., Evans, R.J., Savkin, A.V., and Petersen, I.R. (1999). Stability results for switched controller systems. Automatica, 35(4), 553 - 564.

Zhao, Z.Y., Tomizuka, M., and Isaka, S. (1993). Fuzzy gain scheduling of PID controllers. IEEE Transactions on Systems, Man, and Cybernetics, 23(5), 1392-1398. 